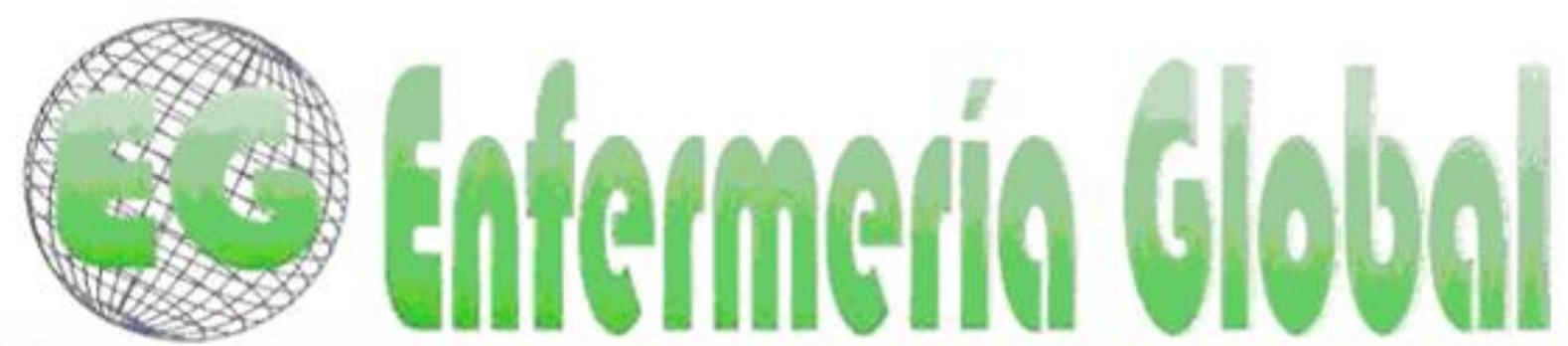

\title{
Calidad de vida profesional de enfermería en urgencias de un hospital de referencia
}

Professional quality of life of nurse in a hospital emergency unit reference

\section{${ }^{*}$ Fernández Araque, Ana ${ }^{* *}$ Cuairán Sola, Miriam ${ }^{* * *}$ Curbelo Rodríguez, Rafael}

*Doctora en Enfermería. Docente Grado de Enfermería Universidad de Valladolid. E-mail: afa@enf.uva.es **Graduada en Enfermería. Servicio de Salud de Aragón. Zaragoza. ${ }^{* * *}$ Doctor en Medicina. Docente Universidad de Valladolid. España.

Palabras clave: Calidad Vida Profesional; enfermería; urgencias; motivación intrínseca; apoyo directivo Keywords: Quality professional; nursing; emergency; intrinsic motivation; managerial support

\section{RESUMEN}

Objetivo: El objetivo de este estudio fue medir la Calidad de Vida Profesional de los enfermeros en la unidad de urgencias de un hospital en su puesto de trabajo, a través de tres dimensiones: motivación intrínseca, apoyo directivo y cargas de trabajo. La mayoría de estudios sobre calidad de vida a nivel mundial se hacen principalmente en colectivos de enseñanza y también los profesionales de la salud, sufren desgaste profesional, según estudios diversos. Si queremos mejorar la calidad de los cuidados prestados, debemos empezar conociendo cómo percibe el profesional de Enfermería dicha calidad de vida, así como si existen o no factores que la condicionen.

Métodos: Estudio descriptivo transversal con análisis correlacional. El presente estudió investigó la calidad de vida profesional de los enfermeros de una Unidad de Urgencias de un Hospital público español. Fue llevado a cabo entre Marzo y Mayo de 2014. Para ello se utilizó el "cuestionario CVP-35".

Resultados: La tasa de respuesta fue alta. Los enfermeros percibieron una baja calidad de vida profesional. La calidad de vida profesional se correlaciona con el Apoyo directivo y la Motivación intrínseca, aunque no se asocia con la Carga de Trabajo. La edad es la variable sociodemográfica que influye en cómo perciben su calidad de vida profesional los enfermeros de urgencias.

Conclusión: En relación con otros estudios, si mejoramos el Apoyo directivo, la percepción de la calidad de vida profesional de los profesionales de enfermería que trabajan en urgencias aumentaría. 


\section{ABSTRACT}

Objective: The objective of this study was to measure the quality of professional life of nurses in their workplace in the emergency unit of a Hospital, through three dimensions intrinsic motivation, support and management workloads. Most studies on quality of life worldwide are made primarily in teaching groups and health professionals. According to various studies, this group is very likely to suffer burnout. If we improve the quality of care provided, we must start knowing how nursing professionals perceive quality of life, as well as whether there are constraining factors involved.

Methods: Cross-sectional study survey of nurses working in an Emergency Unit of a Spanish public hospital. It was conducted between March and May of 2014. The sample consisted on 60 subjects. Data were collected using the CVP-35 questionnaire.

Results: The response rate was high. The nurses perceived low quality of professional life. The professional quality of life correlates with management support and intrinsic motivation, although no association with the workload was found. Age is the demographic variable that influences how they perceive their quality of working life emergency nurses.

Conclusion: Compared to other studies, if we improve the management support, perception of the quality of working life of nurses working in emergency increase.

\section{INTRODUCCIÓN}

La calidad de los servicios prestados por las Organizaciones Sanitarias está directamente relacionada con la satisfacción de los profesionales que en ellas trabajan ${ }^{1}$. Según el Comité Técnico Internacional de Enfermería ${ }^{2}$ la carga de trabajo no sólo afecta a los profesionales de Enfermería, sino que de manera indirecta también afecta a los pacientes, disminuyendo la calidad del cuidado prestado. Conocer la Calidad de Vida Profesional (CVP) es un punto de partida para saber qué se podría corregir dentro de estas Instituciones, mejorando la salud laboral de los profesionales y por consiguiente la atención al paciente y los cuidados que éste recibe.

La CVP la definimos como el "Sentimiento de bienestar que se deriva del equilibrio que el individuo percibe asociado a la carga de la profesión y los recursos psicológicos, organizacionales y relacionales de que dispone para afrontarlos" ${ }^{3}$. La importancia de la satisfacción laboral y la calidad de vida profesional son conceptos discutidos universalmente. Diversos autores ${ }^{4-5}$ han valorado la importancia y el carácter innovador de este concepto a nivel mundial, así como sus limitaciones. Por lo tanto entender la salud mediante estudios que midan la satisfacción, y la calidad de vida de los trabajadores es fundamental para vigilar y fomentar la salud laboral, especialmente en los profesionales de enfermería que trabajan con pacientes en situaciones de urgencia en cualquier país ${ }^{6}$.

Entre las herramientas utilizadas para analizar la calidad de vida profesional se encuentra el CVP- 35 (QVP-35 denominación original). Este cuestionario es anónimo, realiza una medida multidimensional de la CVP mediante tres dimensiones: apoyo directivo (AD), carga de trabajo (CT) y motivación intrínseca (MI). Validado por Cabezas (2000) para la población española general y validado posteriormente en entornos del sistema de salud español ${ }^{7}$. Diferentes autores ${ }^{8-10}$ lo han utilizado para medir la calidad de vida profesional en personal sanitario, por considerarlo de fácil aplicación, comparabilidad y con el que se consigue índices de respuesta aceptables. 
La CVP se ha medido principalmente en España en profesionales sanitarios de Atención Primaria (AP), destacando el realizado en AP de Madrid ${ }^{9}$, que mostró una CVP significativamente superior en enfermeras/os (CVP de 6,13 para IC 95\% 5,94 a 6,32) que en médicos y auxiliares de enfermería (4,99 a 5,36 y 5,17 a 5,77 respectivamente); también mostraron diferencias significativas, donde el aumento de edad se relacionaba con una mayor percepción de la Calidad de Vida Profesional. No encontraron relación entre la CVP y el sexo, la localización del lugar de trabajo (urbano/rural), la experiencia en el Centro de Salud o la situación contractual.

Otro estudio realizado en AP de Soria Sur ${ }^{1}$ mostró una puntuación media de 5,68 para la CVP percibida por enfermeras/os y que ante la percepción de un mayor Apoyo directivo y Motivación intrínseca su CVP aumentaba significativamente, no ocurriendo lo mismo con la Carga de trabajo. En 2012 un estudio publicado ${ }^{10}$ en un Hospital internacional, concluyó que las correlaciones de mayor significación se establecían entre la CVP de enfermeras/os y la Motivación intrínseca $(r=0,60)$, y en menor medida con la Carga de trabajo $(r=0,37)$ y el Apoyo directivo $(r=0,36)$. Otros autores $^{11,12}$ inciden en la importancia de estas variables hasta el punto de afirmar que el Síndrome de Burnout, estaría condicionado en mayor medida por la falta de Apoyo directivo y de Motivación intrínseca más que por la Carga de trabajo.

Conocer la CVP de los profesionales en el ámbito de la salud es crucial por la atención directa y requerida constantemente por el paciente y su familia, especialmente en los profesionales de enfermería y en beneficio de su salud laboral, además los profesionales tienden a dar calidad al cliente en la misma medida en que perciben que son tratados por la organización en que trabajan ${ }^{13}$. La realización de este estudio puede resultar fundamental, ya que la motivación laboral es el resultado de una serie de relaciones entre la motivación intrínseca, el apoyo directivo y la carga de trabajo en una unidad tan dinámica como es el servicio de urgencias de un hospital de referencia, estudios que se acompañan de otros que miden el Síndrome de Burnout, paso posterior a la prevención del mismo ${ }^{14}$.

Por todo lo anterior, se plantea la realización de este estudio cuyo objetivo es determinar la CVP percibida por los profesionales de enfermería del servicio de urgencias del Hospital "Miguel Servet" de Zaragoza. Así como valorar si existe relación entre el Apoyo Directivo, la Carga de Trabajo o la Motivación Intrínseca de estos profesionales con respecto a la Calidad de Vida Profesional percibida.

\section{MATERIAL Y MÉTODOS}

Es un estudio descriptivo transversal con un análisis correlacional, llevado a cabo en la Unidad de Urgencias del Hospital "Miguel Servet" de Zaragoza, entre Marzo y Mayo de 2014. Los participantes en el estudio han sido 60 enfermeros/as de dicha unidad, siendo criterio de inclusión tener turno rotatorio (mañana/tarde), sin desglose por grupos. Los profesionales de Enfermería del turno de noche disponen de turno fijo y no rotan.

El instrumento utilizado fue CVP-35, cuyas características métricas fueron analizadas en el año $2003^{8}$, concluyendo en dicho estudio, que el CVP-35 mantiene sus propiedades métricas en entornos diferentes y obtiene una medida multidimensional de la CVP. El cuestionario fue entregado en la unidad, junto con un consentimiento informado, por la investigadora, en ambos turnos y en distintos días durante tres semanas, para aquellos que podían estar ausentes en los distintos días de entrega. 
El cuestionario consta de 35 ítems que se responden en una escala tipo Likert (1-10) donde los valores cuantitativos tienen su equivalencia cualitativa (1-2: nada; 3-5: algo; 6-8: bastante y 9-10: mucho). Los ítems se agruparon en tres indicadores: apoyo directivo (AD), correspondiente a las preguntas del cuestionario: 3,4,5,10,11,14, 16, 20, 22, 23, 28 y 30; carga de trabajo (CT) que se corresponde con las preguntas: 1, 6, $7,8,15,17,18,19,21,24,25$ y 33; y por último motivación intrínseca (MI) que hace referencia a las preguntas: $2,9,12,13,26,27,29,31,32,35$. La pregunta 34 corresponde como tal a Calidad de Vida Profesional global percibida y no queda agrupada dentro de ninguna de las anteriores dimensiones.

Los datos fueron registrados y analizados para su posterior explotación mediante el Programa estadístico IBM SPSS v.21. Primero se procedió al análisis descriptivo mediante parámetros de centralización y dispersión. Para analizar la relación entre las dimensiones del estudio y la CVP (variable dependiente) se realizó un análisis de Correlación de Pearson, la t de Student para la CVP y los diferentes grupos de edad, y el análisis de la varianza para analizar la influencia de las variables sociodemográficas y laborales (variables independientes) sobre la CVP global percibida.

Para la realización del estudio se ha tenido en cuenta la Ley Orgánica 15/1999 de 13 de Diciembre de Protección de datos de carácter personal. Además de solicitar los permisos pertinentes a la Comisión de Investigación de la Unidad de Calidad (UCA) del Hospital "Miguel Servet".

\section{RESULTADOS}

Del total de respuestas $n=60$, un $21,7 \%(n=13)$ de los encuestados eran hombres mientras que un $78,3 \%(n=47)$ eran mujeres. Si analizamos la "edad del encuestado", el grupo de mayor frecuencia era el de los individuos de entre 31 y 40 años $(45,0 \%, n=27)$. En cuanto a la variable "estado civil" se ha visto que el grupo predominante es el de los casados $(56,7 \%, n=34)$. En lo referente al "número de hijos", el valor predominante en la población encuestada es 0 hijos $(55,0 \%, n=33)$.

En relación al "tipo de contrato" (fijo-jornada completa, fijo-tiempo parcial, eventualjornada completa o eventual-tiempo parcial), más de la mitad de las personas encuestadas tiene un contrato fijo $(55,0 \%, n=33)$. Analizando el "lugar de residencia", se puede observar que el $83,3 \%$ de los encuestados vive en Zaragoza capital, no realizando grandes desplazamientos. En la variable que hace referencia al "Tiempo desde que finalizaron sus estudios de Enfermería", el grupo representado con una mayor frecuencia es el de 10 a 20 años $(46,7 \%, n=28)$.

Por último si analizamos la "Experiencia previa en Urgencias" así como los "Años trabajados en este servicio de Urgencias", observamos que la mayoría de los encuestados sí que tienen experiencia previa en una Unidad de Urgencias (66,7\%, n $=40$ ) y además predominan los enfermeros/as que llevan de 6 a 10 años trabajando en la Unidad de Urgencias de este Hospital $(30,0 \%, n=18)$, siendo los que llevan menos de 6 meses un porcentaje muy pequeño de la población encuestada $(3,3 \%, n$ $=2$ ).

En la tabla I se detalla el análisis descriptivo de las 3 dimensiones del estudio $(A D, C T$ y MI) así como de la CVP global percibida. Sobre un total de 10 puntos, la valoración media sobre la Calidad de Vida Profesional global percibida es de 3,97 (DE 1,82; IC 
$95 \% 3,49$ a 4,44). En relación con las tres dimensiones valoradas, se encuentra que el "Apoyo directivo" tiene una puntuación media de 4,57 (DE 1,20; IC 95\% 4,26 a 4,88); la "Carga de trabajo" muestra una puntuación media de 6,94 (DE 0,83; IC 95\% $6,72$ a 7,15$)$; y por último la "Motivación intrínseca" está valorada con una media de 7,40 (DE 0,89; IC 95\% 7,17 a 7,63).

En el análisis de la varianza, sólo se encontraron diferencias significativas (p 0,016 < 0,05 ) en la Calidad de Vida Profesional global para los diferentes Grupos de edad. Para analizar qué "grupo de edad" presenta las diferencias significativas, utilizamos la prueba de t de Student (Figura 1), que nos muestra que sólo el grupo de edad que va desde los 31- 40 años presenta diferencias significativas con el grupo de edad de 41 50 años en cuanto a la valoración de la "CVP" global percibida $(p=0,001<0,05)$.

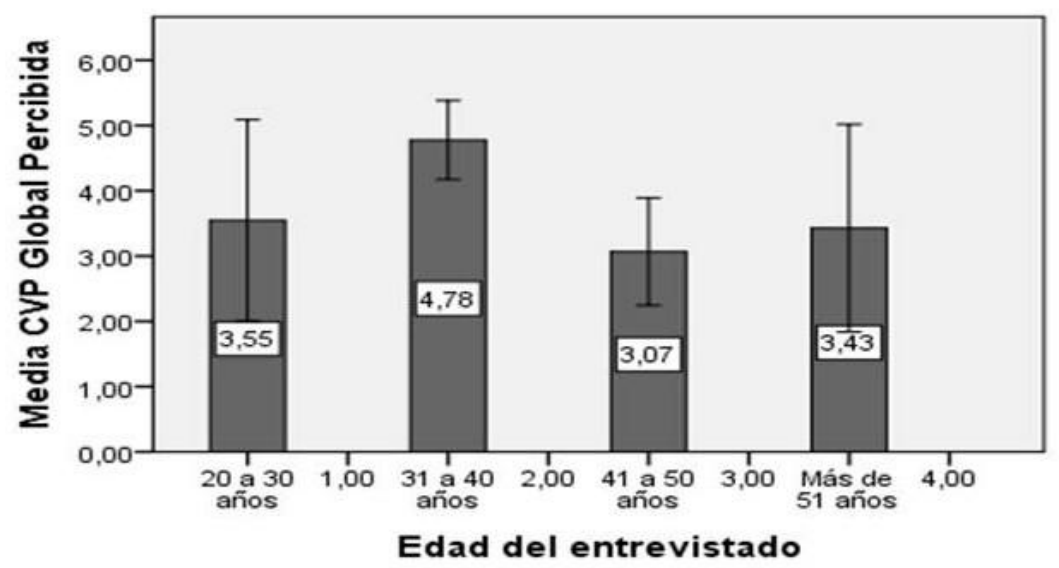

Figura 1: Diagrama de barras de la media en la CVP global percibida para los diferentes grupos de edad. Intervalo de confianza del $95 \%$.

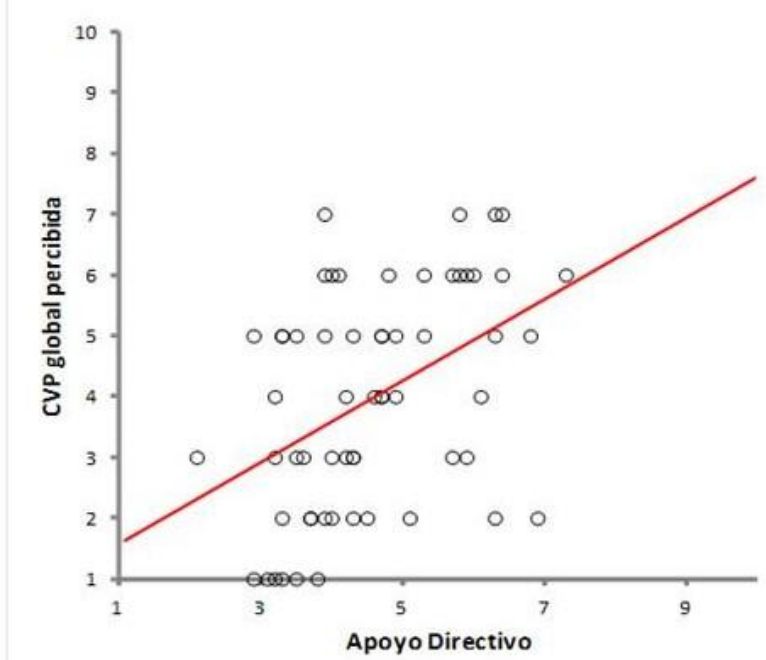

Figura 2: Correlación de Pearson entre la CVP global y el Apoyo directivo $\left(r 0,443, r^{2} \quad 0,196 ; p 0.001<0.05\right.$ y CVP 0,6735). 
La correlación de Pearson (Figura 2), establece que existe relación significativa entre el "AD" y la CVP ( $p=0,000 ; r=0,443)$; así como entre la "Ml" y la CVP ( $p=0,022$; $r=0,296)$; no existiendo tal relación entre la "Carga de trabajo" y la CVP global $(p=0,116 ; r=-0,205)$. Respectivamente (Figura 3 y 4$)$.

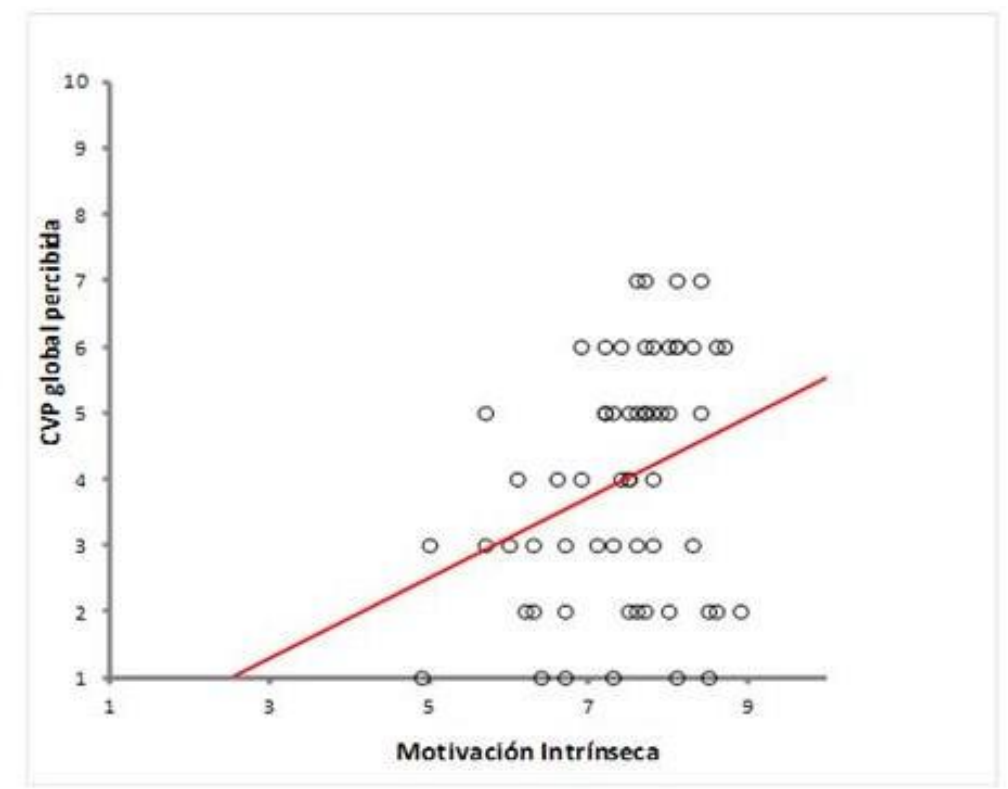

Figura 3: Correlación de Pearson para la CVP global y la Motivación intrínseca ( $\mathrm{r} 0$, $296, r^{2} \quad 0,022$, p $0,022<0,05$ y CVP 0,6075 ).

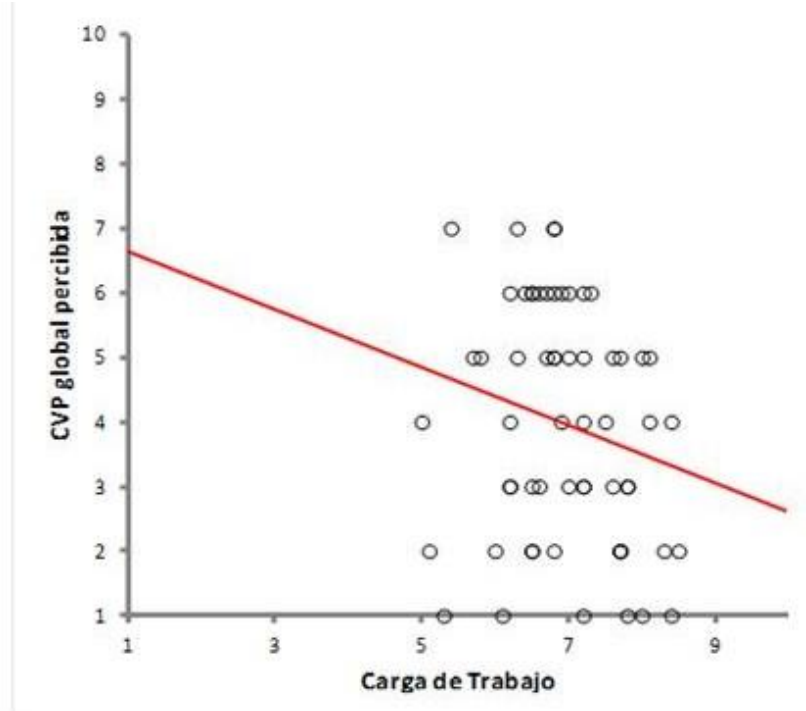

Figura 4: Correlación de Pearson entre la CVP global y la Carga de trabajo (r 0, 205 , $r^{2} 0,042 ; p 0,116>0,05$ y CVP 0,4482 ).

\section{DISCUSIÓN}

Los estudios publicados sobre calidad de vida profesional percibida en profesionales de la salud evidencian suficientemente la fácil aplicación, fiabilidad y comparabilidad del instrumento. El porcentaje de respuesta en nuestro estudio es ligeramente superior $(75 \%)$ al de otros estudios ${ }^{8,10,15-17}$, aunque no en todas las publicaciones 
consultadas exponen la tasa de respuesta. La CVP percibida por los profesionales de enfermería en urgencias del hospital "Miguel Servet" de Zaragoza está por debajo de lo percibido por otros profesionales de enfermería en servicios, principalmente de atención primaria ${ }^{1,3}$, sin embargo, son similares a los valorados por enfermeras/os de unidades de urgencias, emergencias y cuidados intensivos ${ }^{6,18-20}$, pudiendo deberse a la mayor carga de trabajo y complejidad de los cuidados que se requieren en estos servicios, factor que nos induce a pensar la importancia de su valoración.

De conformidad con los resultados obtenidos en nuestro estudio podemos afirmar que los profesionales de Enfermería que trabajan en la Unidad de Urgencias de este hospital tienen una CVP que calificamos como "regular", siendo ésta de 3,97 puntos sobre 10. La Desviación Típica 1,82 y el IC 3,49 a 4,44, muestran una baja dispersión entre las respuestas, no existiendo ningún entrevistado que puntúe con más de 7 su CVP.

Otros estudios reportan puntuaciones medias de CVP global percibida notablemente superiores, algunos ejemplos son el estudio realizado en el Área de Salud de la Comunidad de Madrid (España) ${ }^{7}$ con una puntuación de 6,13, en el Hospital General de Mendoza (Argentina) ${ }^{10}$ con una puntuación de 5,95, en Atención Primaria de Soria Sur (España) con una puntuación de 5,68, en el Área 7 de Salud de la Comunidad de Madrid (España) ${ }^{21}$ con una media de 5,45, y 5 en el Área 11 de Madrid ${ }^{8}$. Sí que un estudio del 2005, realizado en un Hospital de Chile ${ }^{19}$ en el que las enfermeras afirmaban tener una CVP de 3,99 puntos, similar al valor hallado en nuestro estudio.

El género predominante en la Unidad de Urgencias es el femenino, lo que concuerda con datos del año 2011 del Instituto Nacional de Estadística que hablan de la existencia de un $84 \%$ de mujeres entre los profesionales de Enfermería, dato que podría explicarse por la tradicional feminización del cuidado del paciente ${ }^{22}$. En cuanto a la experiencia observamos que un alto porcentaje de los encuestados $(66,7 \%)$, posee experiencia en una Unidad de Urgencias, dato que contrasta con la inexperiencia del personal de enfermería, que se asocia a mayores niveles de agotamiento emocional e insatisfacción en el trabajo ${ }^{23}$.

En relación con la variable "Edad del encuestado", la mayoría de los encuestados están dentro del rango de 31 a 40 años, con una representación del $45 \%$ del total de participantes, al igual que ocurre en el estudio de Sosa ${ }^{3}$. La correlación entre la edad y la valoración de la CVP establece que es el grupo de "31 a 40 años" el que manifiesta percibir una CVP global superior al grupo de "41 a 50 años", con medias de 4,78 y 3,07 respectivamente. Esto concuerda con estudios previos, como el llevado a cabo en 2007 en un Hospital de Chile y que arroja resultados similares. Sin embargo, otro estudio realizado en nuestro país contradice nuestros resultados, dado que no se encontraron diferencias significativas para los diferentes grupos de edad ${ }^{1,2,16}$.

Llama la atención, que no encontramos correlación significativa de la CVP con ninguna otra variable sociodemográfica analizada. En variables como "género" o "tipo de contrato", este hecho va en concordancia con otros estudios. Lo mismo sucede para la variable "Años trabajados en el servicio". Pudiéramos pensar, a priori, que a mayor tiempo trabajado en un servicio, se observaría una menor CVP. No obstante ni nuestro estudio, ni estudios previos apoyan esta hipótesis y no reportan diferencias significativas para la CVP en función de los años trabajados en un servicio. La variable "Estado Civil" tampoco ha mostrado diferencias significativas para la CVP entre los grupos "Soltero" y "Casado" (CVP de 3,94 y 4,09 respectivamente). 
La dimensión Apoyo directivo fue valorada en nuestro estudio con una puntuación de 4,57. Al analizar qué ítems han podido condicionar, en mayor medida esta puntuación, encontramos que el personal de Enfermería de Urgencias afirma sentirse tan solo "algo" apoyados por sus jefes $(4,18)$ lo que contrasta con el elevado "Apoyo por parte de sus compañeros" $(7,90)$. Otros ítems que reciben una baja puntuación son los referidos al "Reconocimiento del esfuerzo" $(3,54)$, a la baja "Posibilidad de promoción" $(3,22)$; pero especialmente a la percepción de "La empresa trata de mejorar la calidad de vida del puesto de trabajo" $(2,73)$, siendo esta última, la peor valorada.

La existencia de una correlación positiva entre CVP y el Apoyo directivo $(p<0,001 ; r=$ 0,443 ), nos muestra que aquellos profesionales de enfermería que percibían un mayor $\mathrm{AD}$, percibían también una mejor CVP. Esta asociación significativa también ha sido reportada por los estudios citados anteriormente.

La dimensión Carga de trabajo recibió una valoración media de 6,94 puntos (IC 95\% $6,72-7,15)$. Dentro de los ítems utilizados para valorar esta dimensión, aquel que recibe mayor puntuación $(9,07)$ es el que se refiere a "Cantidad de trabajo que tengo". También es de destacar la elevada puntuación que reciben "Carga de responsabilidad", "Prisas y agobios por falta de tiempo para hacer mi trabajo" y "Estrés", superando todos ellos los 8,50 puntos. Otro aspecto que puede llevar a la reflexión es la percepción de que su trabajo tiene moderadas consecuencias negativas para su salud, con una puntuación de 5,98. Como dato positivo, destacar que el personal apenas percibe conflictos con otras personas en su trabajo (puntuado con 3,38). Puede que la Carga de trabajo sea la dimensión con mayores diferencias, pudiendo depender ésta del tipo de servicio en el que se trabaja, así como del volumen de pacientes atendidos. En relación a la CT, podríamos pensar que debería existir una correlación significativa negativa entre ésta y la CVP, de manera que al aumentar la cantidad de trabajo sobre una persona, disminuiría su CVP. Sin embargo, los resultados de nuestro estudio demuestran que no existe tal correlación lineal significativa $(p=0,116>0.005)$, y no podemos afirmar que la Carga de trabajo condicione la CVP; ocurre lo mismo en un estudio de Atención Primaria de Soria de 2007.

La Motivación intrínseca fue valorada con la puntuación más alta $(7,40)$. Este dato se debe a que la mayoría de los ítems que hacen referencia a esta dimensión fueron valorados como positivos o muy positivos, con puntuaciones superiores a 6,5; siendo los ítems con peor puntuación "Lo que tengo que hacer queda claro" y "Satisfacción con el tipo de trabajo" con puntuaciones de 6,02 y 6,37 respectivamente.

Coincidimos con los estudios de Albanese, Garrido y Alonso ${ }^{10,17,21}$ en la similar puntuación media otorgada en esta dimensión así como en la asociación positiva entre la CVP y la MI.

Llama nuestra atención que pese a las valoraciones negativas que se hicieron por parte de los profesionales de Enfermería sobre el bajo Apoyo directivo y la elevada Carga de trabajo, los profesionales mantengan una buena Motivación intrínseca para afrontar su labor como enfermeros/as. La valoración del "Estrés" en el puesto de trabajo con una alta puntuación (media 8,57) pudiera ser un condicionante a la hora de prevenir síndrome de Bournout ${ }^{12}$. 


\section{CONCLUSIONES}

Los profesionales de Enfermería del estudio perciben como "regular" su calidad de vida profesional. Pese a ello, disponen de una alta Motivación intrínseca, reforzada, por el elevado apoyo que reciben de sus compañeros y familiares, así como por el sentimiento de estar orgullosos de su trabajo y la capacitación para su desempeño. Existe relación significativa entre la CVP percibida y la Motivación intrínseca así como con el Apoyo directivo. No encontramos relación entre la CVP percibida y la Carga de trabajo. La edad, ha sido la única variable sociodemográfica analizada que influye en la percepción de la CVP.

\section{REFERENCIAS}

1. Fernández A. M. y col. "Calidad de vida profesional de los profesionales de enfermería en atención primaria de Soria", Biblioteca Las Casas-Fundación Index. 2007; 3:70-78.

2. Consejo Internacional de Enfermería (CIE). Las Enfermeras: una fuerza para el cambio. Hojas Informativas "La Enfermería importa". Ginebra: International Council of Nurses; 2014. 70 p.

3. Sosa, O.R., Cheverría, S., Rodriguez, M.E. Calidad de vida profesional del personal de enfermería. Rev. Enferm. Inst. Mex. Seguro Soc. 2010; 18: 153158.

4. Cimete, G., Gencalp, S., Keskin, G. Quality of Life and Job Satisfaction of Nurses. Journal of Nursing Care Quality. 2003; 18: 151-158.

5. Gountas, S., Gountas, J., Soutar, G. Mavondo, F. Delivering good service: personal resources, job satisfaction and nurses? ¿customer? (patient) orientation. Journal of advanced nursing. 2014; 70: 1553-1563.

6. Carrillo, C, Martínez, ME, Vivo,MC, Quiñonero, M, Gómez, R, Celdrán-Gil F. Job satisfaction of medical emergency mobile team and the Coordinating Office of Emergency 061. Rev Calid Asist . 2014; 29: 341-9.

7. Martín Fernández J, Gómez Gascón T, Martínez García-Olalla C, del Cura González MI, Cabezas Peña MC, García Sánchez S. Medición de la capacidad evaluadora del cuestionario CVP-35 para la percepción de la calidad de vida profesional. Aten Primaria. 2008; 40:327-36.

8. Martín J, Cortés JA, Morente M, Caboblanco M, Garijo J, Rodríguez A. Características métricas del Cuestionario de Calidad de Vida Profesional (CVP35). Gac Sanit. 2004; 18:129-36.

9. Martin, J., Gomez, T., Beamud, M., Cortes, J.A., Alberquilla, A. Professional quality of life and organizational changes: a five-year observational study in Primary Care. BMC Health Serv Res. 2007; 4: 101.

10. Albanese, S.,Garelli V. Calidad de vida percibida en el personal de Enfermería de un Hospital General. Rev. de Clínica Neuropsiquiátrica. 2012; 18: 52-59.

11. García, S., Abadía, M.B., Durán, A., Hernández, C., Bernal, E. España: Análisis del sistema sanitario. Sistemas sanitarios en transición. 2011; 12: 1-269.

12. Garriga, D. Calidad de Vida profesional de las enfermeras dentro del equipo multidisciplinar. Investigación en Enfermería. Hospital Psiquiátrico San Juan de Dios. 2010; Pag. 1-6.

13. Cabezas C. Síndrome de desgaste profesional, estrés laboral y calidad de vida profesional. FMC 1998; 5: 491-492.

14.Sanjuan Estallo Laura, Arrazola Alberdi Oihana, García Moyano Loreto María. Prevalencia del Síndrome del Burnout en el personal de enfermería del Servicio 
de Cardiología, Neumología y Neurología del Hospital San Jorge de Huesca. Enferm. Global [revista en la Internet]. 2014 Oct [citado 2015 Ago 23]; 13(36): 253-264. Disponible en: http://scielo.isciii.es/scielo.php?script=sci_arttext\&pid=S1695$61412014000400013 \&$ Ing=es.

15. Mulet Alberola AM, Martí Gil CM, Mejía Recuero M, Martínez Valdivieso L, Marcos Pérez G, Barreda Hernández D. Calidad de vida profesional del personal de los Servicios de Farmacia Hospitalaria del Servicio de Salud de Castilla-La Mancha. Rev. O.F.I.L. 2015, 25; 2:77-83.

16. Akerjordet, K., Severinsson E. Emotionally intelligence nurse leadership: a literature review study. Journal of Nursing Management. 2008; 16(5), 565-577.

17. Franco Vidal A. Percepción de la calidad de vida en un área sanitaria de Asturias. Atención primaria: Publicación oficial de la Sociedad Española de Familia y Comunitaria, ISSN 0212-6567. 2002; 30:483-489.

18. Cimete G, Gencalp NS, Keskin G. Quality of life and job satisfaction of nurses. J Nurs Cuidado Qual. 2003 abril-junio; 18: 151-8.

19.Sanz Moral, Al. Percepción de la Calidad de Vida en personal de enfermería de Servicio de Urgencias de Valladolid. [Trabajo Fin de Grado]. Facultad de Enfermería. Universidad de Valladolid. 2014. Disponible en: http://uvadoc.uva.es/handle/10324/5434

20.Andrades, L., Valenzuela S. Quality of life associated factors in Chileans hospitals nurses. Rev. Latino-Am. Enfermagem [serial on the Internet]. 2007; 15: $480-486$.

21.Garrido Elustondo, S; García Esquina ,E; Viúdez Jiménez, I; López Gómez ,C; Más Cebrián ,E Ballarín Bardají, M. Estudio de la Calidad de Vida Profesional en trabajadores de Atención Primaria del Área 7 de la Comunidad de Madrid. Revista de calidad asistencial. 2010;25:327-333.

22.Santo,T.B., Oguisso, T., Fonseca, R.M. The professionalization of brazilian nursing in the written media of the end of the nineteenth century: a gender analysis. Rev. Latino-Am. Enfermagem [periódico en Internet]. 2011; 19: 12651271.

23.Gutierrez, A.P, Cadela, L.L., Carver, L. The structural relationships between organizational commitment, global job satisfaction, developmental experiences, work values, organizational support, and person-organization fit among nursing faculty. Journal of advanced nursing. 2012; 68: 1553-1563.

Recibido: 23 de agosto 2015; Aceptado: 28 de septiembre 2015

ISSN 1695-6141

() COPYRIGHT Servicio de Publicaciones - Universidad de Murcia 